\title{
Nanoscale Organization of Fullerenes by Self-Assembly in a Diblock Copolymer Host Matrix
}

\author{
Bruno Schmaltz, Martin Brinkmann, and Claude Mathis* \\ Institut Charles Sadron, 6 rue Boussingault, 67083 Strasbourg, France \\ Received July 30, 2004; Revised Manuscript Received September 6, 2004
}

\begin{abstract}
This study illustrates the principle of a novel route to organize $\mathrm{C}_{60}$ on the nanoscale. The method we propose uses the self-assembled structure of a host polystyrene- $b$-polyisoprene (PS- $b$-PI) copolymer as a scaffold to organize polystyrene stars with a $\mathrm{C}_{60}$ core, $\mathrm{C}_{60}(\mathrm{PS})_{f}(f=2,4$, and 6). The effect of the molecular architecture of the stars (size of the PS arms and functionality $f$ ) on the microstructures formed in the blends with a symmetric PS- $b$-PI copolymer $(30 \mathrm{k} / 30 \mathrm{k})$ has been investigated by transmission electron microscopy, differential scanning calorimetry (DSC) and small-angle X-ray scattering (SAXS). The molar mass $M_{\mathrm{w}}{ }^{*}$ and the functionality $f$ of the stars are two key parameters that control their solubilization in the host copolymer matrix, hence, the microstructures and the $\mathrm{C}_{60}$ organization in the blends. Different regimes of solubilization have been identified as a function of the ratio $M_{\mathrm{w}}{ }^{*} / M_{\mathrm{w}}{ }^{\mathrm{PS}}$ between the molar mass of the stars and the PS block of the copolymer $M_{\mathrm{w}}{ }^{\mathrm{PS}}$. Homogeneous and localized solubilization regimes are evidenced for $\mathrm{C}_{60}(\mathrm{PS})_{6}$ stars with $M_{\mathrm{w}} * / M_{\mathrm{w}}{ }^{\mathrm{PS}} \leq 0.35$ and $0.6 \leq M_{\mathrm{w}} * / M_{\mathrm{w}}{ }^{\mathrm{PS}} \leq 2$, respectively. In the latter regime, the $\mathrm{C}_{60}$ cores are preferentially anchored in the middle of the PS lamellae. We also demonstrated that the star's functionality $f$, i.e., its compactness, controls the maximum molar mass of the stars at the solubilization limit in the blends: the higher the functionality of the stars, the higher the molar mass of stars that can be solubilized in the PS lamellae of a copolymer. The overall results are illustrated through different phase diagrams that visualize the required conditions to achieve the spatial organization of $\mathrm{C}_{60}$.
\end{abstract}

\section{Introduction}

In the past decades electroactive organic molecules have attracted much attention owing to their versatility and unique electronic properties of interest for the design of optoelectronic and electronic devices including organic light emitting diodes (OLEDs) and organic field effect transistors (OFETs).,1,2 Among all molecular materials, fullerenes, e.g., $\mathrm{C}_{60}$, have emerged as key molecules and are recognized as exceptional electron acceptors. $^{3}$ In the field of organic solar cells, $\mathrm{C}_{60}$ molecules and derivatives have been successfully used. ${ }^{4}$ One of the requirements for the use of fullerene-based molecules in an electronic device is the possibility to organize spatially the acceptor domains on the length scale of the exciton diffusion distance, i.e., a few tens of nanometers. ${ }^{5}$ The main disadvantage of $\mathrm{C}_{60}$ lies in its poor solubility due to very strong aggregation in conventional organic solvents. Accordingly, incorporation of $\mathrm{C}_{60}$ into thin-film-based devices, e.g., solar cells, requires chemical functionalization. In this perspective, various approaches have been pursued, such as functionalization of $\mathrm{C}_{60}$ with mesomorphic dendrimers or with hydrophilic liquid-crystalline addends to form Langmuir-Blodgett (LB) thin films. ${ }^{6-8}$ However, use of LB films on a large scale in microelectronics is difficult and technologies involving polymer processing are a priori more promising. In 1992, Samulski and co-workers reported the first functionalization of $\mathrm{C}_{60}$ with polystyrene chains. ${ }^{9}$ More recently, the possibility of grafting polystyryl-lithium chains (PS-Li) to $\mathrm{C}_{60}$ to form stars with a high degree of control over the molecular architecture (chemical nature, functionality, and length of the grafted arms) has been demonstrated. ${ }^{10,11}$ For instance, $\mathrm{C}_{60}(\mathrm{PS})_{6}$ stars

* To whom correspondence should be addressed. E-mail: cmathis@ics.u-strasbg.fr. can be synthesized with a well-controlled polydispersity (typically 1.05) and the PS chain length can be varied in a wide range (between 1 and $400 \mathrm{~kg} / \mathrm{mol}$ ). Pure diand tetra-adducts can also be prepared by grafting halogen-terminated polystyrene chains onto $\mathrm{C}_{60}$ through atom-transfer radical addition. ${ }^{12}$ In terms of electronic properties, the stars have revealed improved nonlinear optical (NLO) properties over $\mathrm{C}_{60}$, especially concerning their ultrafast NLO response and their increased second hyperpolarizability. ${ }^{13}$

We report here the first results of a new approach toward the spatial organization of $\mathrm{C}_{60}$. Our method combines the self-assembling properties of classical PS$b$-PI block copolymers with both the high solubility of $\mathrm{C}_{60}(\mathrm{PS})_{6}$ stars in organic solvents such as toluene and their miscibility with polystyrene. This study is dedicated to the structure and organization of PS stars with a $\mathrm{C}_{60}$ core in a PS- $b$-PI copolymer matrix. After having demonstrated the possibility of controlling the spatial organization of the $\mathrm{C}_{60}$ stars in a symmetric PS- $b$-PI diblock copolymer matrix with a lamellar microstructure, we will analyze the effect of the molecular architecture of the stars (length of the PS arms and functionality $f$ ) on the microstructures formed in blends. The motivation for studying the effect of the functionality is two-fold. On one hand, it is known that by increasing the number of PS arms grafted onto the $\mathrm{C}_{60}$ core, one progressively loses the acceptor character of $\mathrm{C}_{60} \cdot{ }^{3} \mathrm{On}$ the other hand, the solubilization of stars into the copolymer matrix is expected to depend strongly on the functionality of the stars $f$ since the compactness of the stars depends closely on $f$. It is therefore interesting to evaluate the conditions required to (i) preserve the acceptor character of the $\mathrm{C}_{60}$ and (ii) achieve the best possible spatial organization of the $\mathrm{C}_{60}$. Finally, the effect of lamellar swelling by $\mathrm{C}_{60}(\mathrm{PS})_{6}$ stars will be 
quantified in order to understand the mechanism of swelling of the PS lamellae by star-shaped objects.

\section{Experimental Section}

Materials. Toluene free of protonic impurities was distilled directly into the apparatus from a red solution of 3-methyl1,1-diphenylpentyllithium. Styrene and isoprene (Aldrich) were distilled over sodium. For anionic polymerization, these monomers were further distilled in ampules equipped with break seals from a $n$-butyllithium solution (prepolymerization). To remove impurities present in commercial fullerene, $\mathrm{C}_{60}$ (>99\% from SES Research) was stirred several hours in pure $\mathrm{THF}$ and recovered by centrifugation. This procedure was repeated until the THF stayed colorless. The $\mathrm{C}_{60}$ was then dried under high vacuum $\left(<10^{-5}\right.$ Torr $)$ at $100-150^{\circ} \mathrm{C}$ and kept and handled under argon in a glovebox. sec-Butyllithium $(s-\mathrm{BuLi})$ was prepared by reacting 2 -chlorobutane with lithium metal in cyclohexane and its concentration determined by titration. 1-Phenylethyl bromide (Aldrich), $\mathrm{CuBr}$ (98\%, Aldrich), and 2,2'-bipyridine (Aldrich) were used as received.

Anionic polymerization was conducted in glass apparatus sealed under high vacuum using the "break-seal" technique. The PS- $b$-PI diblock copolymers are prepared at room temperature in toluene using sec-butyllithium as initiator. The polystyrene block was polymerized first, and after removing an aliquot for characterization, isoprene was added. Finally, the reaction was terminated with methanol. The six-arm polystyrene stars were prepared in two steps. First, polystyrene-lithium was synthesized in toluene using sec-butyllithium as initiator, and then $\mathrm{C}_{60}$ was added in a 1:8 ratio vs PS-Li to ensure the formation of six-arm stars. The ungrafted polystyrene chains were removed by polymer fractionation to get the pure $\mathrm{C}_{60}(\mathrm{PS})_{6}$

Atom-Transfer Radical Polymerization. The ATRP of styrene was conducted in bulk in sealed glass tubes. The styrene, 1-phenylethyl bromide, $\mathrm{CuBr}$ ( 1 equiv), and 2,2 bipyridine ( 2 equiv) mixture was degassed three times; the tube was sealed under vacuum and finally heated at $110{ }^{\circ} \mathrm{C}$. After $2-30 \mathrm{~h}$, the tubes were opened, the remaining monomer removed by distillation, and the polymer dissolved in THF. The greenish solution was centrifuged at $20000 \mathrm{rpm}$ for at least $30 \mathrm{~min}$ to separate the copper salts from the polymer solution. After precipitation in methanol, the polymer was recovered and redissolved in THF. The solution was centrifuged a second time to get rid of the last traces of copper salts. Finally, a white PS-Br was obtained.

Atom-Transfer Radical Addition. Toluene, Br-terminated polystyrene ( $10 \mathrm{wt} \%), \mathrm{CuBr}$ ( 5 equiv), $2,2^{\prime}$ bipyridine (10 equiv), and $\mathrm{C}_{60}$ were introduced in a glass tube. The fraction of $\mathrm{PS}-\mathrm{Br} / \mathrm{C}_{60}$ was 1 for the production of diadducts $\mathrm{C}_{60}(\mathrm{PS})_{2}$ and 6-8 for that of tetra-adducts $\mathrm{C}_{60}(\mathrm{PS})_{4}$. The purple mixture was degassed three times; the tube was sealed under vacuum and heated at $110{ }^{\circ} \mathrm{C}$. After about $60 \mathrm{~h}$, the tube was opened, the toluene removed by distillation, and the polymer dissolved in THF. The brownish solution was centrifuged at $20000 \mathrm{rpm}$ for $0.5 \mathrm{~h}$ to separate copper salts and $\mathrm{C}_{60}$ from the solution. This solution was then precipitated in methanol. The brown precipitate was filtered, washed with methanol, and dried under vacuum.

The various samples were analyzed by size exclusion chromatography (SEC) equipped with a refractive index (RI) detector, a UV-vis detector (set at $320 \mathrm{~nm}$ where only the molecules containing $\mathrm{C}_{60}$ are detected), and a light scattering (LS) detector. As all samples were soluble in THF, this solvent was used as eluent and SEC, equipped with various sets of columns, was previously calibrated using linear PS standards. Since SEC is unable to measure the actual molar masses of a branched architecture; the masses $M_{\mathrm{w}}{ }^{*}$ of the stars were determined using LS. However, due to the strong absorption of light by the fullerenes, these measurements are accurate only when the length of the PS arms becomes large enough to neglect the contribution of the $\mathrm{C}_{60}$ (i.e., $M_{\mathrm{arm}}>10 \mathrm{k}$ for a sixarm star). The characteristics of the block copolymer, the
Table 1. Molecular Characteristics of the PS-b-PI Copolymer and the Various $\mathrm{ST}_{f} M_{\mathrm{w}}$ Stars Determined from the Refractive Index (RI) by SEC and Light Scattering (LS)

\begin{tabular}{|c|c|c|c|c|c|}
\hline \multirow{2}{*}{\multicolumn{2}{|c|}{ sample }} & \multicolumn{3}{|c|}{ SEC (RI) } & \multirow{2}{*}{$\frac{\mathrm{LS}}{M_{\mathrm{w}} \times 10^{3}}$} \\
\hline & & \multirow{2}{*}{$\frac{M_{\mathrm{n}} \times 10^{3}}{26.8}$} & \multirow{2}{*}{$\frac{M_{\mathrm{w}} \times 10^{3}}{28.7}$} & \multirow{2}{*}{$\begin{array}{c}\frac{M_{\mathrm{w}} / M_{\mathrm{n}}}{1.07} \\
1.07\end{array}$} & \\
\hline PS- $b$-PI & PS block & & & & 29 \\
\hline $30 \mathrm{k} / 30 \mathrm{k}$ & PS- $b$-PI & 72.8 & 75.8 & 1.04 & 60 \\
\hline \multirow[t]{2}{*}{$\mathrm{ST}_{6} 10$} & arm & 1.8 & 1.9 & 1.05 & \\
\hline & star & 7.2 & 7.7 & 1.07 & \\
\hline \multirow[t]{2}{*}{$\mathrm{ST}_{6} 18$} & arm & 3.0 & 3.2 & 1.05 & \\
\hline & star & 17.4 & 19.0 & 1.09 & \\
\hline \multirow[t]{2}{*}{$\mathrm{ST}_{6} 27$} & arm & 4.4 & 4.7 & 1.06 & \\
\hline & star & 24.5 & 27.0 & 1.1 & \\
\hline \multirow[t]{2}{*}{$\mathrm{ST}_{6} 60$} & arm & 8.8 & 9.3 & 1.06 & \\
\hline & star & 45.7 & 48.9 & 1.07 & \\
\hline \multirow[t]{2}{*}{$\mathrm{ST}_{6} 105$} & arm & 17.6 & 18.3 & 1.04 & 18 \\
\hline & star & 77.5 & 84.8 & 1.09 & 105 \\
\hline \multirow[t]{2}{*}{$\mathrm{ST}_{6} 530$} & arm & 85.7 & 89.7 & 1.05 & 88 \\
\hline & star & 362 & 396 & 1.09 & 536 \\
\hline \multirow[t]{2}{*}{$\mathrm{ST}_{2} 30$} & arm & 12.2 & 16.1 & 1.32 & \\
\hline & star & 28.7 & 36.7 & 1.28 & \\
\hline \multirow[t]{2}{*}{$\mathrm{ST}_{2} 60$} & arm & 23.0 & 28.8 & 1.25 & \\
\hline & star & 56.3 & 67.4 & 1.2 & \\
\hline \multirow[t]{2}{*}{$\mathrm{ST}_{4} 60$} & arm & 11.5 & 15.1 & 1.31 & \\
\hline & star & 54.7 & 59.2 & 1.08 & \\
\hline \multirow[t]{2}{*}{$\mathrm{ST}_{4} 100$} & arm & 23.6 & 28.3 & 1.2 & 26 \\
\hline & star & 74.0 & 86.9 & 1.17 & 100 \\
\hline
\end{tabular}

parent $\mathrm{PS}-\mathrm{Li}$ or $\mathrm{PS}-\mathrm{Br}$ arms, and the various $\mathrm{C}_{60}$-based stars used in this study are collected in Table 1.

Blend Preparation. A casting protocol for blend preparation was designed to reproducibly prepare bulk binary blends. Solutions $(10 \mathrm{wt} \%)$ of the blends PS- $b$-PI $+x \mathrm{C}_{60}(\mathrm{PS})_{6}(x=\mathrm{wt}$ $\%$ ) were prepared in toluene, a nonselective solvent for polystyrene and polyisoprene. To avoid sample oxidation during slow evaporation of toluene, $0.5 \mathrm{wt} \%$ of an antioxidant agent (Vukanox KB) was added to the blends. The samples were left for slow solvent evaporation over 14-21 days in ambient conditions and sheltered from light. Samples were prepared in the form of disks with a diameter of $1 \mathrm{~cm}$ and a thickness of 1-2 $\mathrm{mm}$. Samples were subsequently dried under vacuum $\left(10^{-3}\right.$ mbar $)$ for $3-4$ days. The conventional annealing procedure reported in the literature for PS- $b$-PI copolymers was not applied in the present case given the possibility for thermolysis of the PS chains from the $\mathrm{C}_{60}$ core. ${ }^{14}$ The $\mathrm{C}_{60}(\mathrm{PS})_{f}$ stars are labeled $\mathrm{ST}_{f} M_{\mathrm{w}} *$ where $M_{\mathrm{w}} *$ is the molar mass of the stars (for instance, $\mathrm{C}_{60}(\mathrm{PS})_{6}$ stars with $M_{\mathrm{w}}{ }^{*}=60 \mathrm{~kg} \mathrm{~mol}^{-1}$ are labeled $\mathrm{ST}_{6} 60$ ). In the following, blends are identified by the $\mathrm{ST}_{f} M_{\mathrm{w}}{ }^{*}$ concentration in the blends given in weight percent (wt \%). The main characteristics of the stars are collected in Table 1. The overall volume fractions of PS in the blends were calculated using the following densities of PI and PS at room temperature: $\rho_{\mathrm{PS}}=1.03 \mathrm{~g} / \mathrm{cm}^{3}$ and $\rho_{\mathrm{PI}}=0.86 \mathrm{~g} / \mathrm{cm}^{3}$.

Transmission Electron Microscopy. For TEM characterization, bulk samples of the blends were microtomed at low temperature (approximately $-80^{\circ} \mathrm{C}$ ) using a Leica Ultracut $\mathrm{S}$ microtome with a FCS cryotemperature attachment. The sections of nominal thickness $50-70 \mathrm{~nm}$ were stained with $\mathrm{OsO}_{4}$ vapors. The sections were examined in bright-field mode using a Philips CM 12 transmission electron microscope at 120 $\mathrm{kV}$. In all TEM micrographs the dark regions correspond to osmium-stained polyisoprene domains.

Differential Scanning Calorimetry. The glass transition of the PI microdomains was determined by differential scanning calorimetry (DSC) on a Perkin-Elmer DSC7 differential scanning calorimeter. The calorimeter was calibrated with decane. Typically, 8-12 $\mathrm{mg}$ of the blends was sealed in aluminum pans. Each sample was quenched from room temperature to $-120^{\circ} \mathrm{C}$ and then heated at a rate of $20{ }^{\circ} \mathrm{C} /$ $\min$ to $130{ }^{\circ} \mathrm{C}$ under dry nitrogen. This procedure was repeated three times on each sample. All the blends in this study exhibited two well-defined glass transitions attributed to the PI and PS microdomains. The PS glass transitions were somewhat broader than the transitions observed for PI. The 


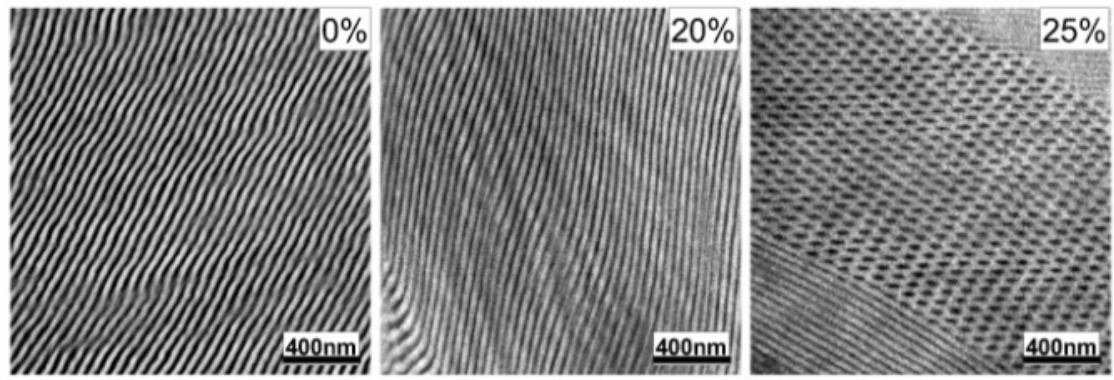

Figure 1. Evolution of the morphology observed by TEM upon blending a PS- $b$-PI (30k/30k) copolymer with $\mathrm{ST}_{6} 10$ stars as a function of increasing wt \% of stars in the blend. Note for the sample with $25 \mathrm{wt} \%$ stars the grain boundary between domains with lying and standing PI cylinders.

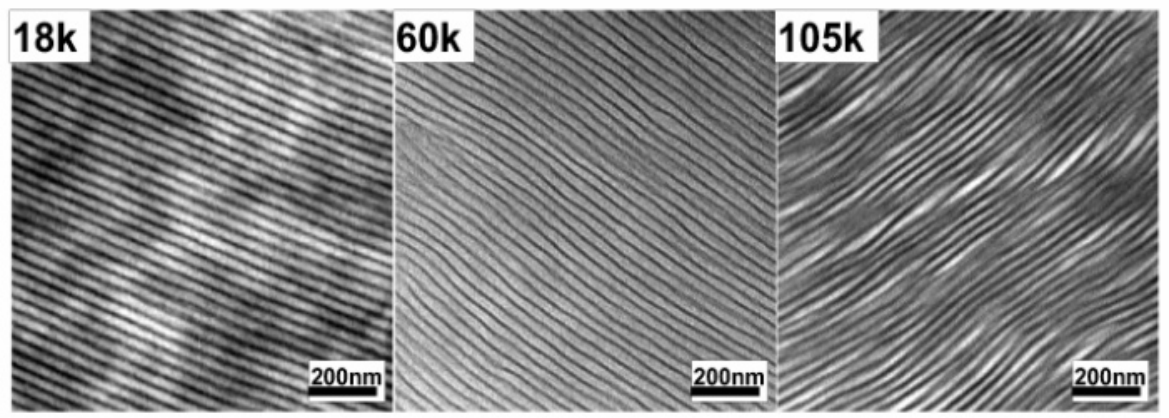

Figure 2. TEM micrographs showing the microstructures formed in blends of a PS- $b$-PI ( $30 \mathrm{k} / 30 \mathrm{k})$ copolymer and $\mathrm{C}_{60}(\mathrm{PS})_{6}$ stars at fixed volume fraction of PS $\phi_{\mathrm{PS}}=0.57$ (20 wt \% of stars) as a function of increasing molar mass of the stars.

glass-transition temperatures were reported as the average inflection point of the three scans. The standard deviation of the $T_{\mathrm{g}}$ values was $\leq 2{ }^{\circ} \mathrm{C}$ for the PI glass-transition temperature.

Small-Angle X-ray Scattering. The lamellar spacings of the binary blends were measured by small-angle X-ray scattering (SAXS) on a Bruker diffractometer equipped with a copper anode $(\mathrm{Cu} \mathrm{K} \alpha, \lambda=0.1542 \mathrm{~nm})$ at an accelerating voltage of $40 \mathrm{kV}$ and a filament current of $40 \mathrm{~mA}$. The scattered intensities were collected on a Highstar (Bruker) twodimensional position-sensitive detector placed $\sim 1 \mathrm{~m}$ from the sample and corrected for wire sensitivity, parasitic scattering, and geometric parameters. The angular calibration of the SAXS system was accomplished with a silver behenate sample.

\section{Results}

We present the main results concerning the microstructures formed in blends of $\mathrm{C}_{60}(\mathrm{PS})_{\mathrm{f}}$ stars with a symmetric PS- $b$-PI copolymer by changing (i) the molar mass $M_{\mathrm{w}}{ }^{*}$ of the $\mathrm{C}_{60}(\mathrm{PS})_{6}$ stars, (ii) the volume fraction of $\mathrm{C}_{60}(\mathrm{PS})_{6}$ in the blends, and (iii) the functionality $f$ of the stars.

(1) Solubilization of $\mathbf{C}_{60}(\mathbf{P S})_{6}$ Stars in the PS Lamellae of the PS- $b$-PI Copolymer. (a) $C_{60}(P S)_{6}$ Stars with $M_{w}{ }^{*} / M_{w}{ }^{P S} \leq 0.35$. Let us first consider the case of small stars with $M_{\mathrm{w}}=10 \mathrm{k}$, i.e., an arm length of $1.8 \mathrm{k}$ much smaller than the PS block of the PS-b-PI copolymer. The sequence of TEM micrographs in Figure 1 depicts the evolution of the morphology upon increasing weight percentage of $\mathrm{ST}_{6} 10$ stars in the blends with the PS- $b$-PI (30k/30k) copolymer. As confirmed by SAXS measurements, introduction of up to $20 \mathrm{wt} \%$ of stars in the blends causes, first, a swelling of the PS lamellae, which can be observed in Figure 1 (typically from 38 to $42 \mathrm{~nm}$ for $10 \mathrm{wt} \%$ stars). Interestingly, for $21 \mathrm{wt} \%$ stars in the blends, we observe a transition from the lamellar microstructure of the PS- $b$-PI copolymer to a hexagonal packing of PI cylinders in a PS matrix with a periodicity of $46 \mathrm{~nm}$ as determined by SAXS. A similar transition to the hex. cyl. phase has been reported in the case of a uniform solubilization of a linear hPS in a lamellar copolymer for homopolymers with small molar mass, e.g., a $2.3 \mathrm{k}$ linear hPS in a PS- $b$-PI (15k/16k). ${ }^{16,17}$ In the present case, this transition occurs at a volume fraction of PS $\phi_{\mathrm{PS}}=0.57$. This value is significantly lower with respect to $\phi_{\mathrm{PS}}=0.67$, corresponding to the reported limit between the lamellar and hex. cyl. phases in the phase diagram of a typical PS- $b$-PI diblock copolymer or blends of a diblock copolymer and a linear hPS. ${ }^{15,17}$ This observation suggests a shift of the domain corresponding to the PI hex. cyl. phase in the phase diagram toward lower values of $\phi_{\mathrm{PS}}$ in blends of $\mathrm{C}_{60}(\mathrm{PS})_{6}$ stars with a symmetric PS- $b$-PI diblock copolymer. The shift of the domain of hex. cyl. in the blends of the PS- $b$-PI copolymer and $\mathrm{ST}_{6} 10$ stars toward lower values of $\phi_{\mathrm{PS}}$ is not clearly understood so far. Further experiments are in progress in order to follow the evolution of the phase diagram in blends involving stars with lower $M_{\mathrm{w}}{ }^{*}$. The microstructures formed in blends with more than 30 wt \% stars could not be examined since samples were extremely brittle and unadapted for cryoultramicrotoming.

(b) $C_{60}(P S)_{6}$ Stars with $0.6 \leq M_{w}^{*} / M_{w}{ }^{P S} \leq 2$. In Figure 2 we depict the microstructures observed by TEM in PS- $b$-PI $(30 \mathrm{k} / 30 \mathrm{k})+\mathrm{C}_{60}(\mathrm{PS})_{6}\left(M_{\mathrm{w}}\right)(20 \mathrm{wt} \%)$ blends for various molar masses of the stars. If the molar masses of the stars lie in the range $0.6 \leq M_{\mathrm{w}} * /$ $M_{\mathrm{w}}{ }^{\mathrm{PS}} \leq 2$, the lamellar microstructure is preserved and no evidence for macrophase separation was found, even for very high wt $\%$ of stars in the blends. In the case of blends with $\mathrm{ST}_{6} 18$ or $\mathrm{ST}_{6} 27$, a wt \% of stars in excess of $60 \mathrm{wt} \%$ brings about the formation of multiple lamellar vesicles. The case of $\mathrm{ST}_{6} 60$ is more peculiar since the lamellar structure is preserved throughout the whole investigated composition range, i.e., up to $80 \mathrm{wt}$ $\%$ stars in the blends. A similar observation has been 


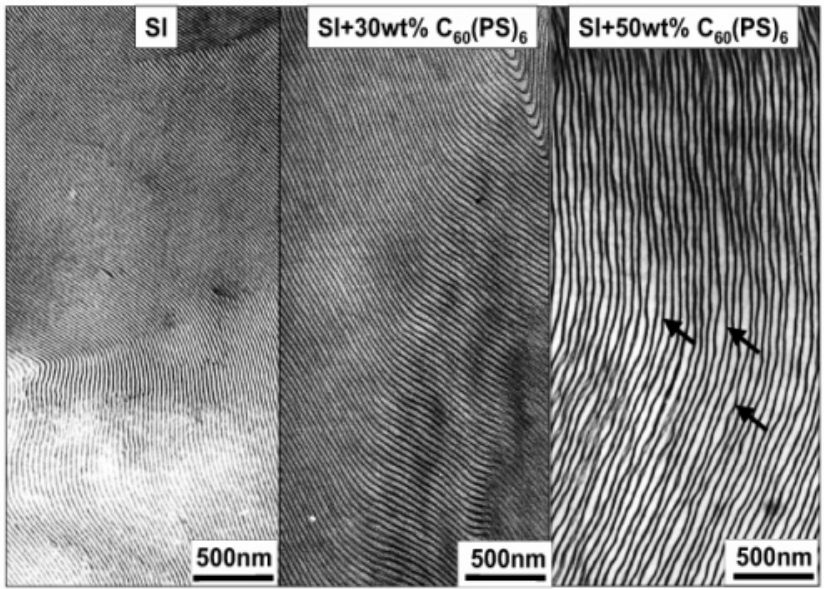

Figure 3. TEM micrographs showing the evolution of the microstructure in binary blends of PS- $b$-PI $(30 \mathrm{k} / 30 \mathrm{k})$ and $\mathrm{ST}_{6} 60$ as a function of increasing volume fraction of stars in the blends. The arrows point to strong fluctuations in the lamellar spacing.

made in blends of PS- $b$-PI copolymer with a linear hPS when the molar masses of the hPS and PS block coincide. ${ }^{18}$

As a matter of fact, for binary systems based on $\mathrm{ST}_{6} 18, \mathrm{ST}_{6} 27$, and $\mathrm{ST}_{6} 60$, we did not observe the transition to the hex. cyl. microstructure when increasing the wt $\%$ of stars up to $\phi_{\mathrm{PS}}=0.89$, i.e., beyond the value of 0.67 which corresponds to the limit between lamellae and hex. cyl. domains in the phase diagram of a classical PS-b-PI diblock copolymers. ${ }^{15}$ Nor did we observe macrophase separation of stars in the blends (80 wt \% stars, $\phi_{\mathrm{PS}}=0.89$ ). In contrast, the TEM micrographs in Figure 3 reveal large fluctuations of the lamellar periodicity as the wt \% of stars in the blends increases. It should be noted that this type of morphology is observed throughout the whole sample. This type of fluctuating lamellar morphology resembles that observed by Kinning et al. in samples of a polystyrene$b$-polybutadiene copolymer (10k/65k) blended with 87 wt $\%$ of a linear $2.1 \mathrm{k}$ hPS. ${ }^{19}$

The lamellar structure is confirmed by SAXS, which shows the typical peak sequence at $q^{\prime}, 2 q^{\prime}$, and $3 q^{\prime}$ with $q^{\prime}$ the scattering vector defined as $q^{\prime}=2 \pi / \lambda$ with $\lambda$ being the total lamellar periodicity. As seen in Figure 4, for blends of $\mathrm{ST}_{6} 60$ and PS- $b$-PI, the characteristic peaks of the lamellar structure are shifted to smaller values of $q$ as the amount of stars in the blends increases. This shift of $q^{\prime}$ indicates that the lamellar spacing $\lambda$ increases with increasing wt \% of stars in the blends. The same tendency is observed for all stars with $0.6 \leq M_{\mathrm{w}} * / M_{\mathrm{w}} \mathrm{PS}$ $\leq 2$ up to 50 wt $\%$ of stars (vide infra). In the SAXS pattern of Figure 4, we observe two interesting points. First, we see that the adjunction of stars in the blends results in the emergence of the second-order peak (which is almost absent for the pure copolymer) and fading of the third-order reflection. This is fully consistent with the fact that even-ordered peaks, which are known to be almost absent in a symmetric lamellar system, will emerge upon increasing the compositional asymmetry, i.e., upon swelling of the PS lamellae by the stars. ${ }^{17}$ Second, we also observe fading of the higher order peaks upon increasing wt \% of stars in the blends. We propose that this fading is partly due to the increase of disorder observed by TEM (fluctuations in lamellar periodicity). However, a structure factor analysis of the peak inten-

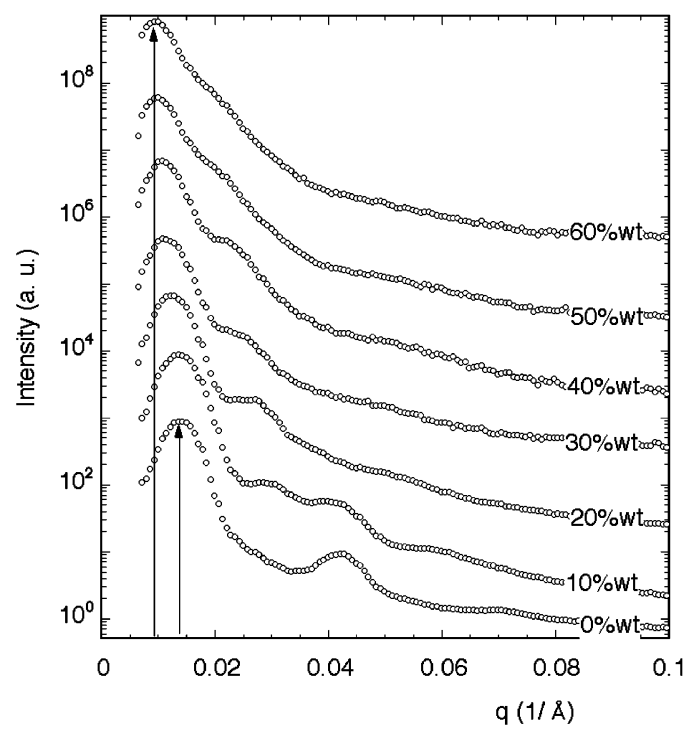

Figure 4. Evolution of the SAXS pattern in blends of PS- $b$ PI (30k/30k) with $\mathrm{ST}_{6} 60$ as a function of increasing wt \% of stars in the blends. For the sake of clarity, successive traces have been shifted along the ordinate axis.

sity variation upon increasing asymmetry of the system including fluctuations in the lamellar spacing would be necessary in order to address this point fully. The important fluctuations of the lamellar spacing are indicative of the inhomogeneous distribution of $\mathrm{C}_{60}$ stars in the PS lamellae as observed in the TEM micrographs of Figure 3.

DSC measurements indicate that the glass-transition temperature $\left(T_{\mathrm{g}}\right)$ of polyisoprene is not significantly affected upon incorporation of stars in the PS- $b$-PI copolymer. The observed values of $T_{\mathrm{g}}$ remain in the range $-53{ }^{\circ} \mathrm{C} \leq T_{\mathrm{g}} \leq-56{ }^{\circ} \mathrm{C}$. Similar to the case of homopolymer blending, these results indicate that $\mathrm{C}_{60}(\mathrm{PS})_{6}$ stars with $0.6 \leq M_{\mathrm{w}} * / M_{\mathrm{w}}{ }^{\mathrm{PS}} \leq 2$ cause a selective swelling of the PS lamellae of the PS- $b$-PI copolymer. ${ }^{16,17}$ Accordingly, the $\mathrm{C}_{60}$ moieties are preferentially confined in the PS lamellae. We thus demonstrate the possibility of organizing the $\mathrm{C}_{60}$ cores on the nanoscale by selective solubilization of the stars in the PS lamellae of the PS$b$-PI copolymer. The detailed dependence of the swelling on the wt \% of stars in the blends will be described in section IV.

The fact that the PS- $b$-PI copolymer can be charged up to 50 wt $\%$ in stars with $0.6 \leq M_{\mathrm{w}}{ }^{*} / M_{\mathrm{w}}{ }^{\mathrm{PS}} \leq 2$ while preserving the lamellar structure implies that we can organize spatially significant amounts of $\mathrm{C}_{60}$ on the nanoscale. For instance, a 50 wt \% blend of $\mathrm{ST}_{6} 18$ with PS- $b$-PI $(30 \mathrm{k} / 30 \mathrm{k})$ corresponds to a proportion of approximately $2 \mathrm{wt} \%$ of $\mathrm{C}_{60}$ in the sample. A large amount of $\mathrm{C}_{60}$ stars in the blends also implies that distances as small as a few nanometers between nearest-neighbor $\mathrm{C}_{60}$ cores can be reached. This could be of interest for potential applications implying charge transport.

(c) $C_{60}(P S)_{6}$ Stars with $M_{w}{ }^{*} / M_{w}{ }^{P S} \geq 3.5$. For stars with $M_{\mathrm{w}}{ }^{*}=105 \mathrm{k}$, SAXS shows that swelling of the PS$b$-PI lamellar structure is limited to about $10 \mathrm{wt} \%$. Interestingly, we observe the presence of fluctuations in the lamellar spacing already for $10 \mathrm{wt} \%$ stars in the blends. As seen in Figure 2, these fluctuations are randomly distributed in the samples and are not related to local plastic deformations such as undulations or chevrons formed by applying mechanical stress to the sample. ${ }^{20}$ The maximum increase of the lamellar width 


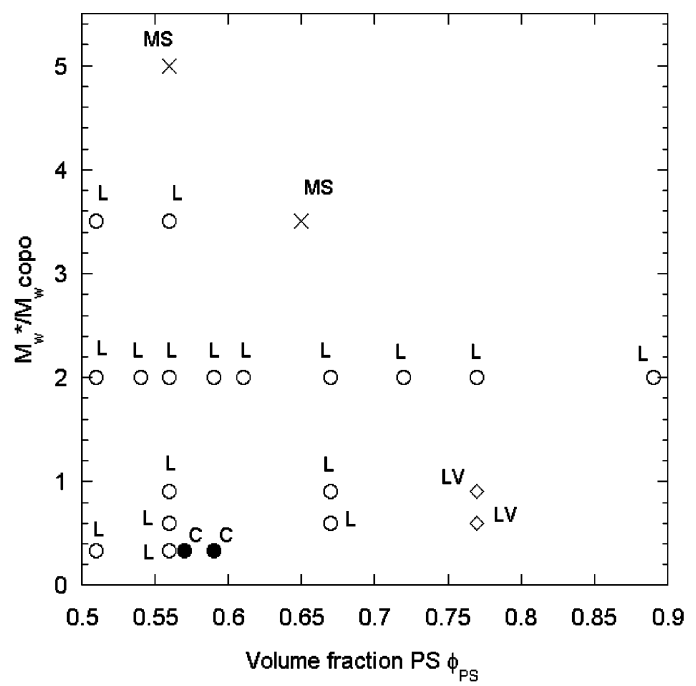

Figure 5. Phase diagram for blends consisting of a symmetric PS- $b$-PI $(30 \mathrm{k} / 30 \mathrm{k})$ copolymer and $\mathrm{C}_{60}(\mathrm{PS})_{6}$ stars of various molar masses $M_{\mathrm{w}}{ }^{*}$ : L, lamellae (open circles); C, hexagonal cylinders of PI (full circles); MS, macrophase separation (crosses); LV, lamellar vesicles (open diamonds).

$(\Delta \lambda)$ is about $1-2$ times the initial width of the PS lamellae of the copolymer. Since the gyration radius in a $\theta$ solvent for $\mathrm{ST}_{6} 105$ star amounts to approximately $6 \mathrm{~nm},{ }^{21}$ the magnitude of $\Delta \lambda$ corresponds to $2-4$ times the diameter of a single $\mathrm{ST}_{6} 105$ star. In the direction parallel to the lamellar plane, the fluctuations are characterized by a curvature of the PS/PI interface with a typical radius on the order of a few hundreds of nanometers, i.e., much larger than the curvature radius at the PS- $b$-PI interfaces in the cylindrical or gyroid morphologies. The occurrence of such fluctuations of the PS lamellar width will be discussed in section IV.

For higher molar masses such as $M_{\mathrm{w}}{ }^{*}=530 \mathrm{k}$, we observe a total phase separation of the stars and the PS- $b$-PI copolymer regardless of the wt $\%$ of stars in the blends. This observation can be explained by the fact that the gyration radius of the stars (calculated for a $\theta$ solvent) amounts to approximately $13 \mathrm{~nm}$, i.e., the diameter of the stars exceeds the width of the PS lamellae of the sole copolymer.

The overall results of TEM and SAXS measurements on the microstructure formed in blends involving $\mathrm{ST}_{6} M_{\mathrm{w}}{ }^{*}$ stars and a symmetric PS- $b$-PI (30k/30k) copolymer are summarized in the phase diagram of Figure 5. For ease of comparison with results obtained on blends with linear hPS, ${ }^{16,17}$ we decided to use the relative molar mass of stars with respect to that of the PS block of the copolymer $M_{\mathrm{w}}{ }^{*} / M_{\mathrm{w}}{ }^{\mathrm{PS}}$.

(2) Solubilization of $\mathrm{C}_{60}(\mathrm{PS})_{2}$ and $\mathrm{C}_{60}(\mathrm{PS})_{4}$ Stars. In Figure 6 we compare the morphologies observed in blends of the PS- $b$-PI (30k/30k) copolymer with $20 \mathrm{wt}$ $\%$ of stars with two and four PS arms and increasing molar mass. The blends with $\mathrm{ST}_{2} 30$ and $\mathrm{ST}_{4} 60$ exhibit the typical swollen lamellar morphology, which is indicative of a preferential solubilization of stars in the PS lamellae of the copolymer. Instead, a very distinct morphology is observed for $\mathrm{ST}_{2} 60$ and $\mathrm{ST}_{4} 100$. Typically, we observe in the latter samples elongated structures consisting of PS inclusions with a narrow size distribution and a mean size of sections perpendicular to the lamellar planes of approximately $500 \mathrm{~nm} \times 100 \mathrm{~nm}$. It has to be stressed that this type of morphology is distinct from the fluctuating lamellar morphology ob-

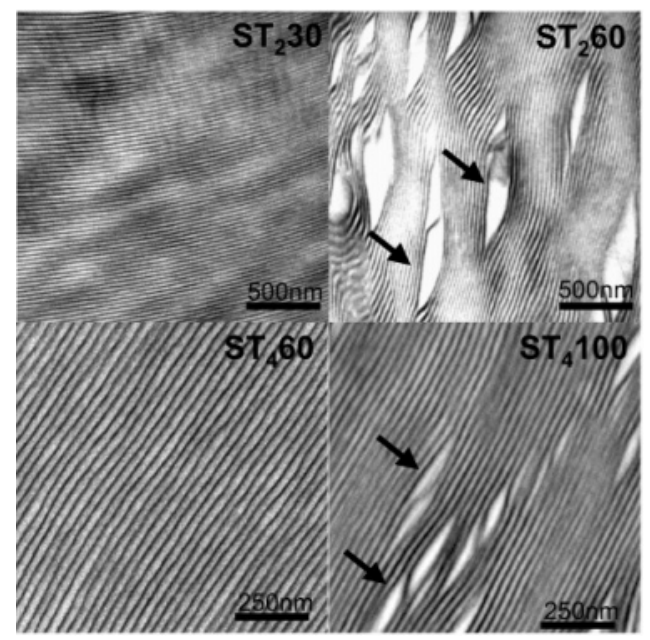

Figure 6. Evolution of the microstructures observed in blends of a symmetric PS- $b$-PI copolymer $(30 \mathrm{k} / 30 \mathrm{k})$ with $20 \mathrm{wt} \%$ of $\mathrm{C}_{60}(\mathrm{PS})_{f}$ stars with $f=2$ and 4 as a function of increasing $M_{\mathrm{w}}{ }^{*}$. The arrows point at mesoscopic inclusions of $\mathrm{C}_{60}(\mathrm{PS})_{f}$ stars.

served in blends with large wt $\%$ of $\mathrm{ST}_{6} 60$ (Figure 3). As a matter of fact, the PS inclusions tend to be associated and are sometimes separated by a few lamellae of the copolymer. For blends with $\mathrm{ST}_{2} 60$ and $\mathrm{ST}_{4} 100$, SAXS confirms the absence of swelling of the PS lamellae of the copolymer. The concentration and average size of these inclusions are found to increase by increasing the wt \% of stars in the samples.

To identify the origin of these inclusions, we also prepared blends of the copolymer with a linear PS homopolymer with $M_{\mathrm{w}}^{\text {lin }}=60 \mathrm{k}$. Indeed, the $\mathrm{C}_{60}(\mathrm{PS})_{2}$ stars can be regarded as linear homopolymers with a $\mathrm{C}_{60}$ at their center. Interestingly, we observed the build up of the same type of mesostructures in the case of a linear hPS but with a slightly larger average size. This suggests that the origin of these inclusions is related to macrophase separation. ${ }^{17}$ This result is in agreement with previous reports on blends with linear hPS. If we refer to the phase diagram of Winey et al. for blends of a linear hPS and a symmetric PS- $b$-PI, ${ }^{17}$ we find that the composition of the $\mathrm{ST}_{2} 6020 \mathrm{wt} \%$ blend is located at the boundary between swollen lamellae and spatially disordered micelles. However, in the present case, the observed morphology cannot be regarded as disordered micelles.

The comparison between the microstructures formed in blends of a copolymer with $\mathrm{C}_{60}(\mathrm{PS})_{\mathrm{f}}$ stars $(f=2,4$, and 6) nicely illustrates the impact of the star's functionality $f$ on the limit of solubilization. Under the condition $\lambda_{\mathrm{PS}}{ }^{\circ}>2 r_{\mathrm{g}}{ }^{*}$, we observe that the larger the functionality $f$ of the stars, the larger the molar mass of the stars that can be solubilized in the PS lamellae of the copolymer without inducing macrophase separation.

\section{Discussion}

(1) Homogeneous vs Localized Solubilization of $\mathbf{C}_{60}(\mathbf{P S})_{6}$ Stars. The present observations show that the degree and homogeneity of the solubilization of $\mathrm{C}_{60}(\mathrm{PS})_{6}$ stars in the PS lamellae of the PS- $b$-PI copolymer is a function of both molar mass $M_{\mathrm{w}}{ }^{*}$ and functionality $f$. The effect of the molar mass of the linear homopolymer hPS on its solubilization in a PS- $b$-PI matrix has been extensively studied by Hashimoto et al. as well as Winey et al. ${ }^{16,17}$ The miscibility of a linear homopolystyrene 
a)

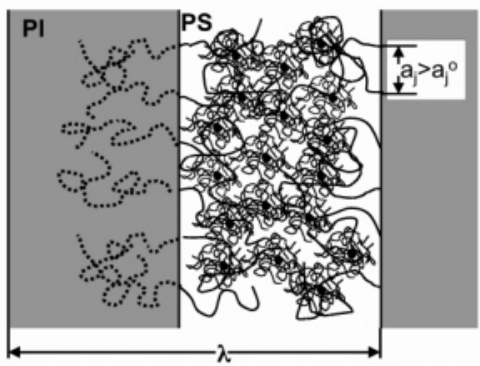

b)

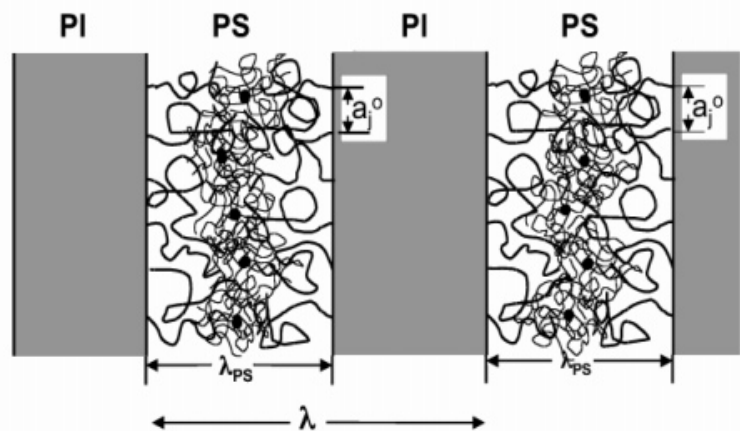

Figure 7. Schematics of the $\mathrm{C}_{60}(\mathrm{PS})_{6}$ star distribution in the PS lamellae of the PS- $b$-PI copolymer (30k/30k): (a) homogeneous solubilization for $M_{\mathrm{w}} *=10 \mathrm{k}$; (b) Localized solubilization of the stars for $18 \mathrm{k} \leq M_{\mathrm{w}}{ }^{*} \leq 60 \mathrm{k}$, illustrating the anchoring of the $\mathrm{C}_{60}$ cores in the center of the PS lamellae and the periodic organization of $\mathrm{C}_{60}$ every second lamellae.

(molar mass, $M_{\mathrm{w}}$ lin) in the PS domains of a lamellar copolymer was found to decrease with increasing $M_{\mathrm{w}}$ lin . Hashimoto et al. evidenced two regimes of solubilization: (i) homogeneous solubilization for small $M_{\mathrm{w}}$ lin and (ii) localized solubilization for high $M_{\mathrm{w}}$ lin 16 The approach of Winey et al. was similar to that by Hashimoto et al. but involved a continuous distribution of hPS chains in the PS lamellae of the copolymer with essentially a variation of the width of the distribution with $M_{\mathrm{w}}$ lin $.17,18$ Basically, it was observed that hPS with small $M_{\mathrm{w}}$ lin are homogeneously dispersed in the PS lamellae and thus contribute to both axial (perpendicular to lamellar plane) and lateral (parallel to lamellar plane) swelling of the PS lamellae whereas for higher $M_{\mathrm{w}}$ lin hPS chains are rather localized toward the center of the PS lamellae, which undergo mainly axial swelling.

Our TEM and SAXS results suggest a similar phenomenology. Similar to the case of blending with a linear homopolystyrene, we can define two regimes of solubilization as schematized in Figure 7: (i) a regime of homogeneous solubilization of the stars in the PS lamellae of the copolymer (Figure 7a) and (ii) a regime of more localized solubilization (Figure 7b). The case of homogeneous solubilization is characterized by a swelling of the PS lamellae that is followed by a transition to a structure of hex. packed PI cylinders in a PS matrix containing the $\mathrm{C}_{60}(\mathrm{PS})_{6}$ stars. In the case of $\mathrm{ST}_{6} 10$, we observed that the transition to the hex. cyl. phase occurs at a rather low volume fraction of $\mathrm{PS}, \phi_{\mathrm{PS}}=0.57$. In the case of localized solubilization for $\mathrm{ST}_{6} M_{\mathrm{w}}{ }^{*}$ with $0.6 \leq M_{\mathrm{w}} * / M_{\mathrm{w}}{ }^{\mathrm{PS}} \leq 2$, we observed that the transition to the hex. cyl. phase was suppressed. Instead, the PS lamellae of the copolymer can harbor a larger amount of stars and cause a progressive swelling up to $\phi_{\mathrm{PS}}=$ 0.77 while preserving the lamellar microstructure. We want to emphasize that the change of microstructure upon blending from lamellar to hex. cyl. is used here as

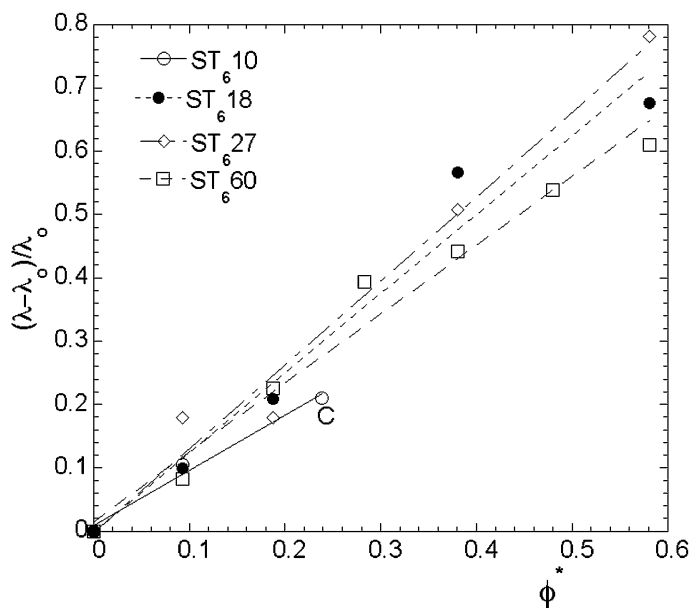

Figure 8. Relative variation of the total lamellar periodicity measured by SAXS with the volume fraction $\phi^{*}$ of various $\mathrm{C}_{60}(\mathrm{PS})_{6}$ stars $\left(\mathrm{ST}_{6} 10, \mathrm{ST}_{6} 18, \mathrm{ST}_{6} 27\right.$, and $\left.\mathrm{ST}_{6} 60\right)$ in binary blends with a PS- $b$-PI copolymer. In the case of $\mathrm{ST}_{6} 10$, we have also shown the periodicity of the cylinder morphology $(\mathrm{C})$. The straight lines are the result of linear fits (see text).

the criterion that distinguishes both homogeneous and localized solubilization regimes.

A further criterion that distinguishes these two regimes of solubilization is the dependence of the PS lamellar width with the volume fraction of $\mathrm{C}_{60}(\mathrm{PS})_{6}$ stars in the blends. In Figure 8 we plotted the relative variation of the total periodicity of the lamellar structure obtained by SAXS measurements for blends containing $\mathrm{ST}_{6} M_{\mathrm{w}} *$ stars $\left(10 \mathrm{k} \leq M_{\mathrm{w}} * \leq 60 \mathrm{k}\right)$ as a function of increasing volume fraction of stars $\phi^{*}$.

Typically, the periodicity of the PS- $b$-PI copolymer, i.e., $38 \mathrm{~nm}$, increases to $64 \pm 4 \mathrm{~nm}$ for $50 \mathrm{wt} \%$ ST60 in the blends. For all stars the total periodicity of the lamellae $\lambda$ scales almost linearly with the volume fraction of stars in the blends $\phi^{*}$ according to the relation $\lambda / \lambda_{\mathrm{o}}=1+\alpha \phi^{*}$. For all samples with $18 \mathrm{k} \leq M_{\mathrm{w}}{ }^{*} \leq 60 \mathrm{k}$, the slope $\alpha$ determined from a linear fit lies in the range $1.1-1.3 \pm 0.1 .^{22}$ In contrast, for stars with $M_{\mathrm{w}} *=10 \mathrm{k}$, the slope $\alpha$ is found to be significantly lower, i.e., $\alpha=$ 0.8 .

The fact that $\alpha<1$ means that the swelling occurs both laterally and axially. This is the case for stars with $M_{\mathrm{w}}{ }^{*}=10 \mathrm{k}$, which supports a regime of homogeneous solubilization. In principle, it is possible to obtain the variation of the mean distance between chemical junction points of the PS- $b$-PI copolymer by simple volumetric considerations. Usually the expansion between chemical junction points at the PS- $b$-PI interface is characterized by the mean distance $a_{j}$ between chemical junction points. $a_{j}, a_{j}^{\circ}, \lambda$, and $\lambda_{o}$ are related through the following equation 16

$$
\lambda / \lambda_{\mathrm{o}}=\frac{\left(a_{j}^{\mathrm{o}}\right)^{2}}{\left(a_{j}\right)^{2}\left(1-\phi^{*}\right)}
$$

where $\lambda_{0}$ and $\lambda$ are the total periodicities of the lamellar structure before and after swelling, $a_{j}{ }^{\circ}$ and $a_{j}$ are the mean distances between chemical junction points at the PS/PI interface before and after swelling, and $\phi^{*}$ is the volume fraction of $\mathrm{ST}_{6} M_{\mathrm{w}} *$ stars in the blend.

In fact, $\alpha \rightarrow 1$ means that the swelling tends to be essentially axial, i.e., the distance between chemical junction points remains constant, the reason for which, $\alpha>1$ in the present case of localized solubilization of 
$\mathrm{C}_{60}(\mathrm{PS})_{6}$ stars with $18 \mathrm{k} \leq M_{\mathrm{w}}{ }^{*} \leq 60 \mathrm{k}$, might be inferred from eq 1 . From this equation it can be easily seen that $\alpha>1$ implies contraction of the distance between chemical junction points at the PS/PI interface. Contraction of the distance between the chemical junction points of the PS- $b$-PI copolymer can be understood only if the PS chain conformation of the copolymer is modified in the blends with respect to the sole PS- $b$-PI copolymer. Indeed, if the PS chains are elongated axially, the requirement for a constant volume density of PS will imply contraction of the chemical junction points at the PS/PI interface.

(2) Limit of Molar Mass to the Solubilization of $\mathrm{C}_{60}(\mathrm{PS})_{6}$ Stars: Role of the Star's Functionality $f$. In the case of a linear homopolymer, the upper limit of the molar mass $M_{w}$ lin to the solubilization in the PS lamellae of the copolymer can be obtained from the phase diagram by Winey et al. ${ }^{17}$ From Figure 4 of ref 17 we obtain the limit between the lamellar and the disordered micelle's morphologies for $\phi_{\mathrm{PS}}=0.56$ at $M_{\mathrm{w}}$ lin/ $M_{\mathrm{w}}{ }^{\mathrm{PS}}=1.7 .{ }^{16}$ However, it is known that stars are more compact than linear hPS for the same molar mass. The star's conformation involves a rather rigid core region around the $\mathrm{C}_{60}$, which is difficult to be accessed by the PS chains of either the copolymer or a neighboring star. The larger the functionality $f$, the larger the compactness of the stars. From the literature we can find the values of the ratio between the gyration radii of the star $R_{g}{ }^{*}$ and of the linear hPS $R_{g}$ lin. $\left(R_{g}{ }^{*}\right)^{2} /\left(R_{g} \text { lin }\right)^{2}=0.45$ and 0.63 for stars with $f=6$ and 4 , respectively. ${ }^{21}$ This higher compactness of the stars with respect to the linear hPS supports our observation, i.e., the fact that stars with a larger molar mass than linear hPS can be solubilized in the PS lamellae of the PS- $b$-PI copolymer. We can determine the upper limit of the star's molar mass $M_{\mathrm{w}}{ }^{*}$ that can be solubilized in the PS lamellae. We make the assumption that it corresponds to a star with a gyration radius equivalent to that of a linear PS with $M_{\mathrm{w}}$ lin $=1.7 M_{\mathrm{w}}{ }^{\mathrm{PS}}$. Using the equations for the gyration radii of the stars and linear hPS in a theta solvent (this is the situation closest to the solid state) as given in the literature ${ }^{21,23}$

$$
R_{g}^{*}=0.034\left(M_{\mathrm{w}}{ }^{*}\right)^{1 / 2} f^{-0.346}
$$

and

$$
R_{g}^{\text {lin }}=0.029\left(M_{w}^{\operatorname{lin}}\right)^{1 / 2}
$$

where $f$ is the functionality of the stars, we can obtain the ratio $\rho$ between $M_{\mathrm{w}}{ }^{\text {lin }}$ and $M_{\mathrm{w}}{ }^{*}$ for $R_{g}{ }^{\text {lin }}=R_{g}{ }^{*}$ at the solubility limit

$$
\rho=\frac{M_{\mathrm{w}}^{*}}{M_{\mathrm{w}} \operatorname{lin}}=\left(0.85 f^{0.346}\right)^{2}
$$

From eq 4 we observe that the ratio $\rho$ clearly increases with increasing $M_{\mathrm{w}}{ }^{*}$ and also that $\rho>1$ for $f \geq 3$. For stars with $f=6$, we obtain $\rho=2.5$, and for $f=4$, we have $\rho=1$.9. Interestingly, for $f=2$, we obtain $\rho \approx 1$, as expected for a linear hPS.

In Figure 9 we depict a phase diagram for constant $\phi_{\mathrm{PS}}=0.56$ (20 wt \% stars in the blends) as a function of both molar mass $M_{\mathrm{w}} *$ of the stars and functionality $f$. We plotted as a continuous line the result of eq 4 , corresponding to the boundary between the domain where the stars are solubilized in the PS lamellae of

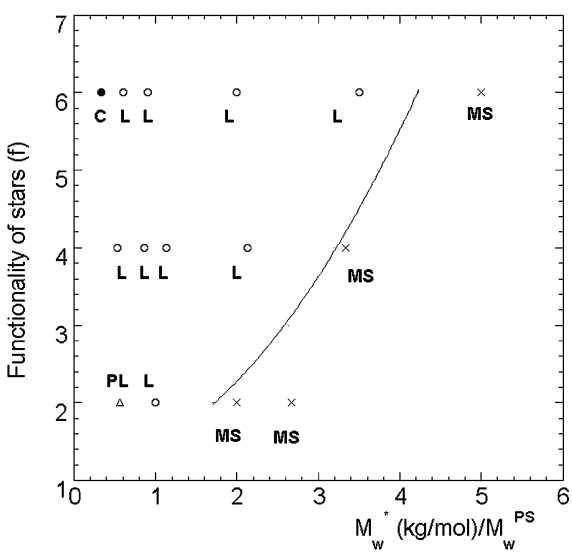

Figure 9. Phase diagram illustrating the dependence of the microstructure on the functionality of the stars $f$ and molar mass $M_{\mathrm{w}} *$ in blends for a constant PS volume fraction $\phi_{\mathrm{PS}}=$ 0.56 . The full line is the result of a fit using eq 4 (see text): $\mathrm{L}$, lamellae (open circles); MS, macrophase separation (crosses); $\mathrm{C}$, hexagonal cylinders of PI (full circles); PL, perforated lamellae (PL).

the copolymer and macrophase separation. We obtain a rather good agreement between the observed morphologies and the predicted domains for ordered microstructures and macrophase-separated morphologies. This agreement clearly illustrates the impact of the star's architecture on their solubilization in a copolymer matrix and hence gives the conditions to achieve the spatial organization of the $\mathrm{C}_{60}$ cores in the copolymer matrix.

(3) Role of Molecular Diffusion on the Organization of $\mathrm{C}_{60}(\mathrm{PS})_{\mathrm{f}}(f=4$ and 6$)$ Stars in a Copolymer Matrix. Our TEM experiments have clearly shown that blends involving $\mathrm{ST}_{6} 60$ or $\mathrm{ST}_{6} 105$ stars show very strong fluctuations of lamellar spacing indicating a strongly inhomogeneous solubilization of the stars in the PS lamellae of the PS- $b$-PI copolymer. The condition for forming ordered architectures based on $\mathrm{C}_{60}$ stars is partly determined by the mobility of the stars in the copolymer matrix. In the case of blends involving a linear hPS, reptation of the linear hPS chains is possible and allows them to find the optimum position and conformation in the melt of the PS arms in the PS lamellae. The situation is different for $\mathrm{C}_{60}(\mathrm{PS})_{6}$ stars since stars with $f \geq 3$ cannot reptate like linear chains of PS. ${ }^{24}$ The diffusion constant $D$ of stars with functionality $f$ and arm length $N$ in a medium of linear chains with the same chemical nature has been determined to follow eq $5^{24}$

$$
D \approx \exp [-k(f-2) N]
$$

where $k$ is a constant.

Following eq 5 the diffusion constant of stars should decrease exponentially with the arm length $N$ at fixed $f$ and should also change significantly with the functionality $f$ at a fixed value of $N$. Accordingly, the fluctuations in lamellar spacing observed for blends with $\mathrm{ST}_{6} 105$ and $\mathrm{ST}_{4} 100$ may have a kinetic origin because of the limited diffusion of stars within the lamellae during the self-organization process of the surrounding copolymer matrix. This argument is further supported by results on the microphase separation in star block copolymer for which the ordering kinetics has been shown to be much slower than for linear diblock copolymers. $^{25}$ 


\section{Conclusions}

This study presents new results concerning the swelling behavior of a symmetric PS- $b$-PI copolymer with stars of the type $\mathrm{C}_{60}(\mathrm{PS})_{f}(f=2,4$, and 6$)$. We evidenced the existence of different solubilization regimes which are determined by the molecular architecture of the stars, i.e., their functionality $f$ and their molar mass $M_{\mathrm{w}}{ }^{*}$. For $\mathrm{C}_{60}(\mathrm{PS})_{6}$ stars, two distinct regimes of solubilization have been identified as a function of the molar mass. Stars with $M_{\mathrm{w}} * / M_{\mathrm{w}} \mathrm{PS} \leq 0.35$ are rather homogeneously distributed in the PS lamellae of the copolymer, and this opens the possibility for a structural transformation from lamellae to a hexagonal packing of PI cylinders. Instead, in blends involving $\mathrm{C}_{60}(\mathrm{PS})_{6}$ stars with $0.6 \leq M_{\mathrm{w}}{ }^{*} / M_{\mathrm{w}}{ }^{\mathrm{PS}} \leq 2$, the swelling occurs in a regime of localized solubilization preserving largely the lamellar microstructure. In this latter regime it is possible to organize spatially the fullerenes on the nanoscale by selective solubilization of the stars in the PS lamellae of a PS- $b$-PI copolymer. We also demonstrated that the star's functionality $f$ determines the maximum molar mass of the stars at the solubilization limit. Whereas the solubilization of $\mathrm{C}_{60}(\mathrm{PS})_{2}$ stars in the PS lamellae of the copolymer is similar to that of a linear hPS, the solubilization of stars with $f \geq 4$ is clearly distinct due to the higher compactness of the stars. It is also suggested that the absence of reptation for stars with $f \geq 3$ affects the kinetics of the organization mechanism of the stars in the copolymer matrix.

This work illustrates an interesting route to the nanoscale organization of electron-acceptor molecules such as $\mathrm{C}_{60}$ by taking advantage of the self-assembling properties of a host structuring matrix like a simple diblock copolymer. Ordered $\mathrm{C}_{60}$ nanostructures may alternatively be produced by directly grafting the selfassembling PS- $b$-PI copolymer onto the $\mathrm{C}_{60}$, hence generating planes and wires of $\mathrm{C}_{60}$. This strategy will be described in a forthcoming communication.

Acknowledgment. We acknowledge Michel Rawiso for fruitful discussions and giving access to the SAXS facility at ICS. Technical support by C. Foussat, R. Meens, A. Rameau, F. Schnell, and S. Zehnacker is also acknowledged.

\section{References and Notes}

(1) Friend, R. H.; Gymer, R. W.; Holmes, A. B.; Burroughes, J. H.; Marks, R. N.; Taliani, C.; Dos Santos, D. A.; Brédas, J.L.; Lögdlund, M.; Salaneck, W. R. Nature 1999, 397, 121.

(2) Dimitrakopoulos, C. D.; Malenfant, R. L. Adv. Mater. 2002, 14,99 .
(3) Xie, Q.; Perez-Cordero, E.; Echegoyen, L. J. Am. Chem. Soc. 1992,114 (10), 3978

(4) Brabec, J. C.; Sariciftci, N. S.; Hummelen, C. Adv. Funct Mater. 2001, 11, 15.

(5) Nelson, J. Curr. Opin. Solid State Mater. Sci. 2002, 6, 87.

(6) (a) Nierengarten, J.-F.; Eckert, J.-F.; Nicoud, J.-F.; Ouali, L.; Krasnikov, V.; Hadziioannou, G. Chem. Commun. 1999, 617 (b) Felder, D.; Gallani, J.-L.; Guillon, D.; Heinrich, B.; Nicoud, J.-F.; Nierengarten, J.-F. Angew. Chem., Int. Ed. 2000, 39, 201.

(7) Nakashima, N.; Ishii, T.; Shirakusa, M.; Nakanishi, T.; Murakami, H.; Sagara, T. Chem. Eur. J. 2001, 7, 1766.

(8) Guillon, D.; Nierengarten, J.-F.; Gallani, J.-L.; Eckert, J.-F ; Rio, Y.; del Pilar Carreon, M.; Dardel, B.; Deschenaux, R. Macromol. Symp. 2003, 192, 63.

(9) Samulski, E. T.; DeSimone, J. M.; Hunt, M. O.; Menceloglu, Y. Z.; Jarnagin, R. C.; York, G. A.; Labat, K. B.; Wang, H.; Chem. Mater. 1992, 4, 1153.

(10) Ederlé, Y.; Mathis, C. Macromolecules 1997, 30, 2546.

(11) Weber, V.; Duval, M.; Ederlé, Y.; Mathis, C. Carbon 1998 36,839 .

(12) (a) Audouin, F.; Nunige, S.; Nuffer, R.; Mathis, C. Synth. Met. 2001, 121, 1149. (b) Audouin, F.; Nuffer, R.; Mathis, C. J. Polym. Sci., Part A: Polym. Chem. 2004, 42, 3456.

(13) (a) Koudoumas, E.; Konstantaki, M.; Mavromanolakis, A.; Couris, S.; Ederlé, Y.; Mathis, C.; Seta, P.; Leach, S. Chem. Phys. Lett. 2001, 335, 533. (b) Venturini, J.; Koudoumas, E.; Couris, S.; Janot, J. M.; Seta, P.; Mathis, C. J. Mater. Chem. 2002, 12 (7), 2071.

(14) Mathis, C.; Nunige, S.; Audouin, F.; Nuffer, R. Synth. Met. 2001, 121, 1153.

(15) Khandpur, A. K.; Förster, S.; Bates, F. S.; Hamley, I. W.; Ryan, A. J.; Bras, W.; Almdal, K.; Mortensen, K. Macromolecules 1995, 28,8796 .

(16) Hashimoto, T.; Tanaka, H.; Hasegawa, H. Macromolecules 1990, 23, 4378. Hashimoto, T.; Tanaka, H.; Hasegawa, H. Macromolecules 1991, 24, 240.

(17) (a) Winey, J. I.; Thomas, E. L.; Fetters, L. J. Macromolecules 1991, 24, 6182. (b) Winey, K. I.; Thomas, E. L.; Fetters, L. J. Macromolecules 1992, 25, 2645.

(18) Shull, K. R.; Winey, K. I. Macromolecules 1992, 25, 2637.

(19) Kinning, D. J.; Winey, K. I.; Thomas, E. L. Macromolecules 1988, 21, 3502

(20) Cohen, Y.; Brinkmann, M.; Thomas, E. L. J. Chem. Phys. 2001, 114, 984.

(21) Grest, G. S.; Fetters, L. J.; Huang, J. S.; Richter, D. Advances in Chemical Physics; Prigogine, I., Rice, S. A., Eds.; John Wiley \& Sons: New York, 1996; Vol. XCIV, p 77.

(22) Note that the periodicities measured by SAXS for the blends with $\phi^{*}>0.48$ are affected by a large uncertainty because of both the absence of well-defined higher order peaks and the increasing breadth of the peaks. However, these uncertainties cannot account on their own for the observed values of $\alpha \geq$ 1.1 .

(23) Schmidt, M.; Burchard, W. Macromolecules 1981, 14, 210.

(24) Grest, G. S.; Fetters, L. J.; Huang, J. S.; Richter, D. Advances in Chemical Physics; Prigogine, I., Rice, S. A., Eds.; John Wiley \& Sons: New York, 1996; Vol. XCIV, p 143 and references therein.

(25) Floudas, G.; Pispas, S.; Hadjichristidis, N.; Pakula, T.; Erukimovich, I. Macromolecules 1996, 29, 4142.

MA048421E 